REVIEW

\title{
The Application of the Monte Carlo Code FLUKA in Radiation Protection Studies for the Large Hadron Collider
}

\author{
Giuseppe BATTISTONI ${ }^{1}$, Francesco BROGGI ${ }^{1}$, Markus BRUGGER ${ }^{2}$, Mauro CAMPANELLA ${ }^{1}$, Massimo CARBONI ${ }^{5}$, \\ Anton EMPL ${ }^{10}$, Alberto FASSÒ $^{16}$, Ettore GADIOLI ${ }^{1}$, Francesco CERUTTI ${ }^{2}$, Alfredo FERRARI ${ }^{2}$, Anna FERRARI $^{14}$, \\ Maria Vittoria GARZELLI ${ }^{15}$, Mattias LANTZ ${ }^{13}$, Andrea MAIRANI ${ }^{1}$, M. MARGIOTTA ${ }^{8}$, Cristina MORONE ${ }^{7}$, \\ Silvia MURARO ${ }^{1}$, Katia PARODI ${ }^{9}$, Vincenzo PATERA ${ }^{6}$, Maurizio PELLICCIONI ${ }^{6}$, Lawrence PINSKY ${ }^{10}$, \\ Johannes RANFT ${ }^{4}$, Stefan ROESLER ${ }^{2, *}$, Sofia, ROLLET ${ }^{12}$, Paola R. SALA ${ }^{1}$, Mario SANTANA ${ }^{3}$, Lucia SARCHIAPONE ${ }^{5}$, \\ Massimiliano SIOLI ${ }^{8}$, George SMIRNOV ${ }^{2}$, Florian SOMMERER ${ }^{9}$, Christian THEIS ${ }^{2}$, Stefania TROVATI ${ }^{2}$, R. VILLARI $^{6}$, \\ Heinz VINCKE ${ }^{2}$, Helmut VINCKE ${ }^{2}$, Vasilis VLACHOUDIS ${ }^{2}$, Joachim VOLLAIRE ${ }^{2}$ and Neil ZAPP ${ }^{11}$

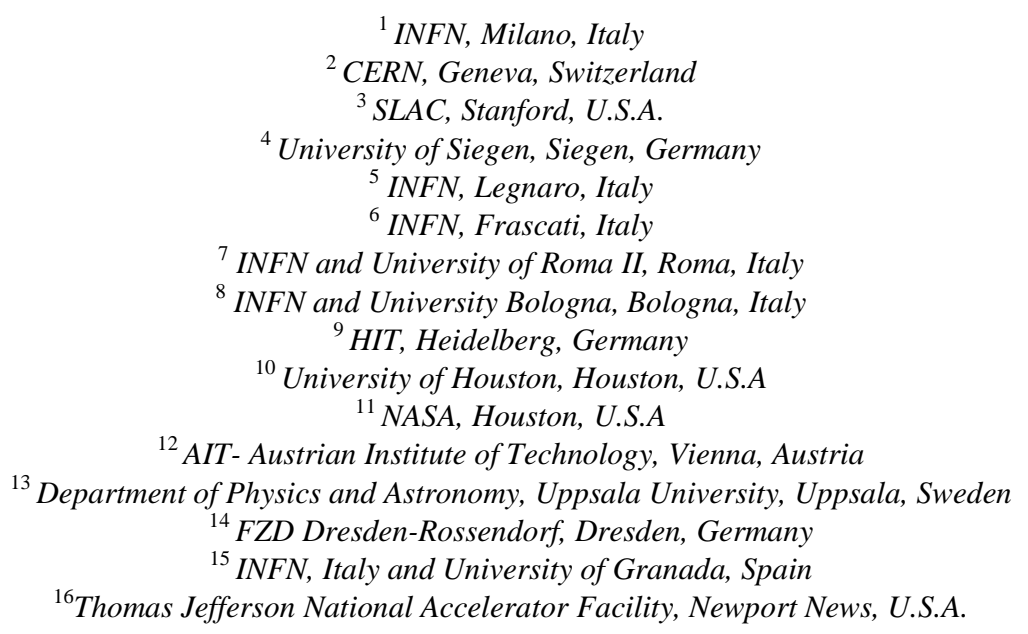

\begin{abstract}
The multi-purpose particle interaction and transport code FLUKA is integral part of all radiation protection studies for the design and operation of the Large Hadron Collider (LHC) at CERN. It is one of the very few codes available for this type of calculations which is capable to calculate in one and the same simulation proton-proton and heavy ion collisions at LHC energies as well as the entire hadronic and electromagnetic particle cascade initiated by secondary particles in detectors and beam-line components from $\mathrm{TeV}$ energies down to energies of thermal neutrons. The present paper reviews these capabilities of FLUKA in sketching the relevant physics models along with examples of radiation protection studies for the LHC such as shielding studies for underground areas occupied by personnel during LHC operation and the simulation of induced radioactivity around beam loss points. Integral part of the FLUKA development is a careful benchmarking of specific models as well as the code performance in actual, complex applications which is demonstrated with examples of studies relevant to radiation protection at the LHC.
\end{abstract}

KEYWORDS: Large Hadron Collider, radiation protection, shielding, activation, FLUKA

\section{The FLUKA Code}

FLUKA is a general-purpose particle interaction and transport code with roots in radiation protection studies at high energy accelerators. ${ }^{1,2)}$ It therefore comprises all features needed in this area of application, some of which are outlined in the following.

The module for hadronic interactions is called PEANUT (PreEquilibrium Approach to Nuclear Thermalization) and consists of a phenomenological description (Dual Parton Model-based Glauber-Gribov cascade) of high-energy interactions (up to $20 \mathrm{TeV}$ ), a generalized intra-nuclear

*Corresponding author, E-mail: Stefan.Roesler@cern.ch cascade, and pre-equilibrium emission models as well as models for evaporation, fragmentation, fission, and de-excitation by gamma emission. Interactions of ions are simulated through interfaces with different codes based on models applicable in certain ranges of energy (DPMJET3 above $5 \mathrm{GeV} /$ nucleon, rQMD-2.4 between 0.1 and $5 \mathrm{GeV} /$ nucleon, Boltzmann Master Equation below $0.1 \mathrm{GeV} /$ nucleon). ${ }^{2)}$ The interface with DPMJET3 $^{3)}$ also allows the simulation of minimum-bias proton-proton and heavy ion collisions at LHC energies which enormously facilitates calculations of stray radiation fields around LHC experiments.

The transport of neutrons with energies below $20 \mathrm{MeV}$ is performed by a multi-group algorithm based on evaluated 
cross section data (ENDF/B, JEF, JENDL, etc.) binned into 260 energy groups, 31 of which are in the thermal energy region. For a few isotopes $(1 \mathrm{H}, 6 \mathrm{Li}, 10 \mathrm{~B}, 14 \mathrm{~N})$ point-wise cross sections can be optionally used during transport. The detailed implementation of electromagnetic processes in the energy range between $1 \mathrm{keV}$ and $1 \mathrm{PeV}$ is fully coupled with the models for hadronic interactions.

Many variance reduction techniques are available in FLUKA, among others, weight windows, region importance biasing as well as leading particle, interaction and decay length biasing.

The capabilities of FLUKA are unique for studies of induced radioactivity, especially with regard to nuclide production, their decay and the transport of residual radiation. Particle cascades by prompt and residual radiation are simulated in parallel based on microscopic models for nuclide production and a solution of the Bateman equations for activity build-up and radioactive decay. The decay radiation and its associated electromagnetic cascade are internally flagged as such in order to distinguish them from the prompt cascade. This allows the user to apply different transport thresholds and biasing options to residual and prompt radiation and to score both independently.

Particle fluence can be multiplied with energy-dependent conversion coefficients to effective dose or ambient dose equivalent $^{4)}$ at scoring time. Prompt and residual dose equivalent can thus be computed in three-dimensional meshes, the latter for arbitrary user-defined irradiation and cooling profiles.

Integral part of the FLUKA code development is benchmarking of new features against experimental data. It includes both the comparison of predictions of individual models to measurement results (e.g., nuclide production cross sections) as well as benchmarks for actual complex situations as, for example, arising during accelerator operation. Thus, the applications for the LHC will be complemented below by benchmark examples.

\section{Radiation Protection Studies for the LHC}

The LHC, presently being commissioned at CERN, will accelerate two counter-rotating beams of protons or ions to a maximum energy of $7 \mathrm{TeV}$ for protons (presently, proton beams are accelerated to $3.5 \mathrm{TeV}$ ) and $2.8 \mathrm{TeV} /$ nucleon for lead ions, respectively. The accelerator has a circumference of about $27 \mathrm{~km}$ and consists of eight octants (see Fig. 1). Four of the octants house the detectors of the experiments ALICE, ATLAS, CMS, and LHCb, two octants serve beam-cleaning (collimation) purposes and the remaining two octants contain the accelerating RF cavities and the beam dumps, respectively. Most of the radiation protection studies for the LHC have relied heavily on FLUKA as it was one of the very few Monte Carlo code available which can reliably simulate hadronic interactions at LHC energies.

The LHC is located underground at depths of $80-100 \mathrm{~m}$ with access to machine areas being prohibited during operation. Exceptions are the counting rooms of the

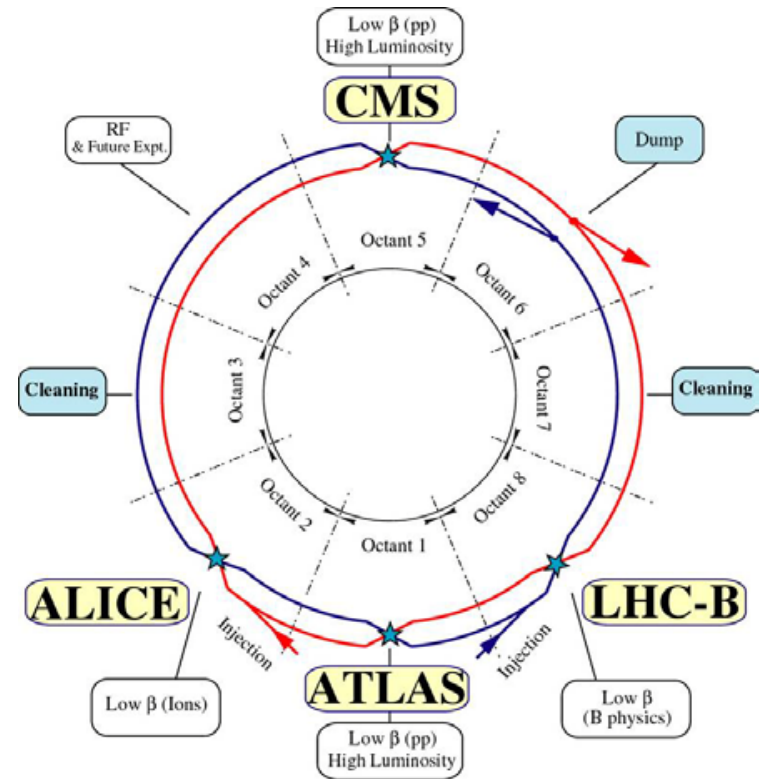

Fig. 1 Schematic layout of the LHC.

experiments, where personnel could be exposed to prompt stray radiation. Thus, they were subject to detailed shielding studies; as an example, the shielding design for the LHCb counting rooms is presented below.

With the LHC being a super-conducting storage ring, particles losses have to be low by design around the sections operated at cryogenic temperatures (more than $80 \%$ of the accelerator). Consequently, activation of accelerator components is concentrated to a limited number of areas, such as the above mentioned collimation and beam dump regions. These areas, however, required detailed assessments of induced radioactivity in order to achieve, at the same time, job dose optimization and limitation of machine down-time for repair and maintenance work. In this context, the capabilities of FLUKA to predict residual dose rates have opened completely new possibilities for a pro-active radiation protection during accelerator design, e.g., the consideration of the later handling of highly radioactive components already in the design phase.

Finally, stray radiation fields around the LHC often consist of neutrons, charged hadrons, electrons and photons having energies from that of thermal neutrons up to several hundreds of $\mathrm{GeV}$. Therefore, the use of standard calibration sources for instruments measuring such radiation fields is not appropriate in many cases and a detector characterization by FLUKA simulation was required.

In the following chapters examples for all three types of radiation protection studies for the LHC (shielding, activation and instrument calibration) will be given.

\section{Shielding Studies}

\section{Attenuation Benchmark}

An attenuation benchmark inter-comparison of the predictions of different Monte Carlo codes, including 

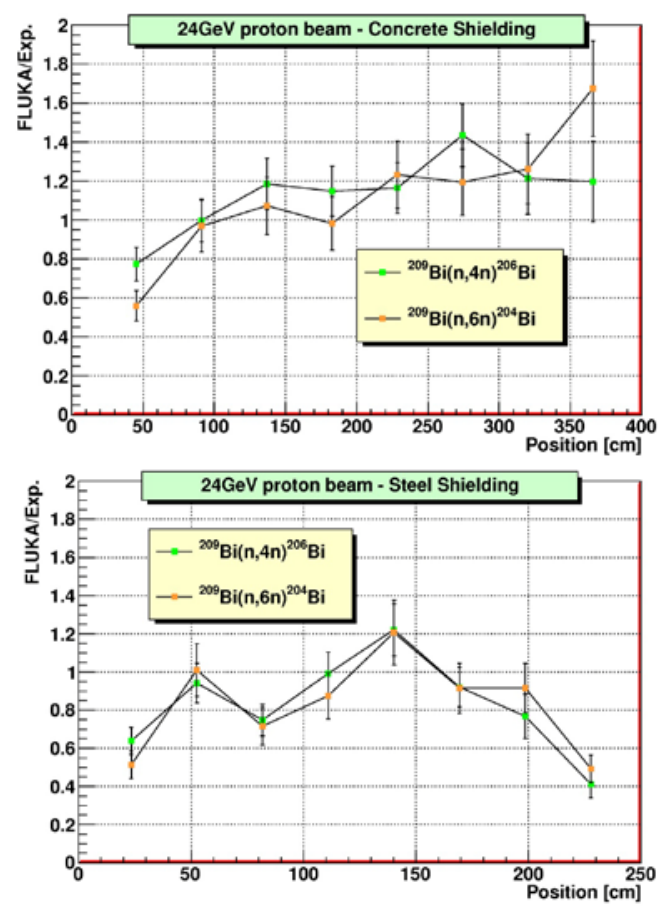

Fig. 2 Ratios of reaction rates predicted by FLUKA and measured at various depths in concrete (top panel) and iron shielding (bottom panel). ${ }^{5,6)}$

FLUKA, with experimental data has been published recently by the SATIF-10 Task Force. ${ }^{5)}$ The experiment, carried out at the Alternating Gradient Synchrotron (AGS) accelerator, consisted in activating Bi-foils behind layers of concrete and steel shielding of different thicknesses which were placed laterally to a mercury target. Two proton beam energies were used, $2.83 \mathrm{GeV}$ and $24 \mathrm{GeV}$, respectively; results for the latter are shown in Fig. 2.,6) The graphs present ratios of calculated and measured reaction rates for the creation of ${ }^{204} \mathrm{Bi}$ and ${ }^{206} \mathrm{Bi}$ nuclides in the foils as function of depth inside the shielding. Details on the experimental setup, such as dimensions and material characteristics, were given by the Task Force in order to ensure well-defined conditions for the benchmark. The provided information also included energy-dependent reaction cross sections which were used to fold calculated neutron fluences. It should be noted that the benchmark explores high energy neutron fluences through the shielding due to the high reaction threshold energies (20-40 MeV). The results demonstrate that FLUKA reproduces the data within $40 \%$ up to several meters of shielding.

\section{LHCb Shielding}

Data readout and acquisition from the LHC detectors requires rather short distances such that all related equipment had to be placed underground in areas accessible by personnel during operation. Therefore, FLUKA was used for shielding studies of all LHC experiments. In case of the $\mathrm{LHCb}$ detector, which was installed in a cavern of the previous LEP collider, a shielding wall had to be designed

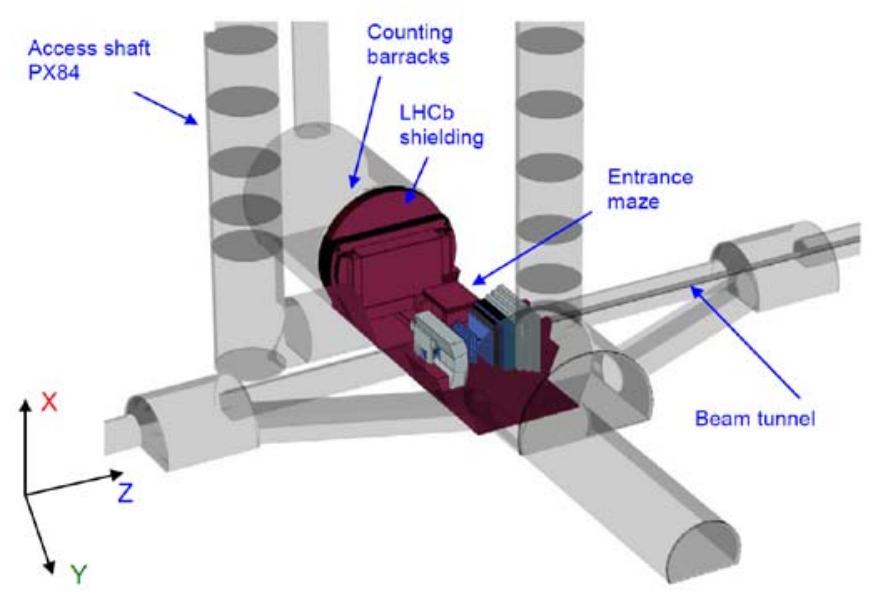

Fig. 3 Layout of the LHCb experimental cavern as implemented in the FLUKA simulations ${ }^{7)}$ and visualized with SimpleGeo. ${ }^{8)}$

and erected in order to separate the counting rooms from the detector area (see Fig. 3). Several constraints rendered the design a complex optimization process: passage of services, such as cables and ducts, a large mobile part to allow the replacement of detector components, an access maze as well as structural and financial considerations.

During this iterative process FLUKA played a central part by allowing a detailed simulation of both particle cascades from accidental beam losses and proton-proton collisions in the entire underground cavern. ${ }^{7)}$ As shown in Fig. 3 the geometry contains a detailed representation of the underground caverns and shafts, the LHCb detector as well as the shield wall with ducts and passages. The studies revealed that, due to the low luminosity of LHCb (a factor of 50 lower than for CMS and ATLAS), an accidental loss of the full LHC beam determines shielding thickness and design rather than stray radiation from proton-proton collisions.

Figure 4 shows a vertical, transverse section through the shield wall in the centre of the cavern. Colors indicate dose equivalent per lost $7 \mathrm{TeV}$ proton; the contour corresponding to $20 \mathrm{mSv}$ (the legal annual dose limit at CERN) for an accidental loss of one full beam at ultimate beam intensity of $4.7 \times 10^{14}$ protons is indicated with a blue arrow above the legend. As can be seen, the limit is respected in the bottom part of the cavern, while it could be exceeded in the upper part to which access is therefore prohibited during operation.

\section{Induced Radioactivity}

\section{Activation Benchmark}

The unique features of FLUKA for the computation of induced radioactivity and residual dose rates mentioned above were extensively benchmarked at the CERF facility. At this facility a positively charged hadron beam of $120 \mathrm{GeV}$ interacts in a copper target creating a stray radiation field which can be used for a large variety of studies, among 


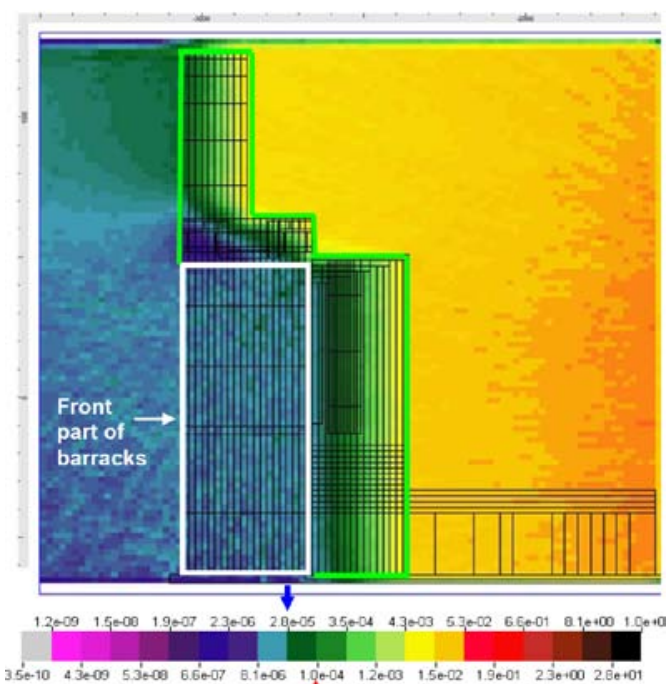

Fig. 4 Spatial distribution of ambient dose equivalent on both sides of the shielding (vertical cross-section through the center of the cavern perpendicular to the beam axis) given in terms of pSv/primary proton. ${ }^{7)}$ Note that the considered ultimate beam intensity is $4.7 \times 10^{14}$ protons.

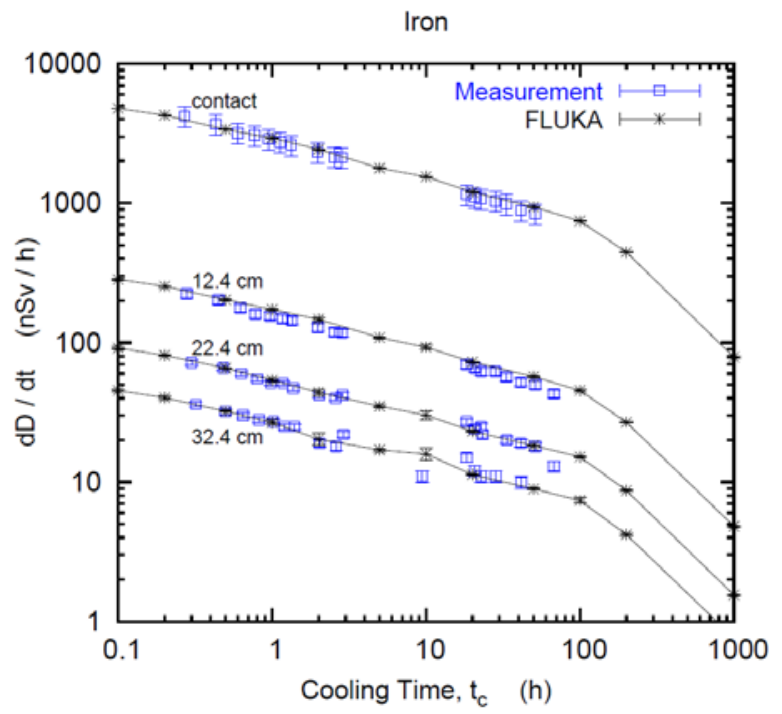

Fig. 5 Residual dose equivalent rates as function of cooling time on contact to an iron sample and at three different distances as measured and as calculated with FLUKA. ${ }^{9)}$

others the activation of material samples. Different materials commonly used for accelerator components and shielding (copper, iron, aluminum, etc.) were irradiated and their activation measured by gamma spectrometry and with dose rate instruments at different cooling times.

Furthermore, the irradiation as well as the radioactive build-up and decay were simulated with FLUKA and results compared to the experimental data. ${ }^{9,10)}$ The benchmark showed that FLUKA predicts specific activities of individual nuclides within $20-30 \%$ in many cases and is also able to reproduce residual dose equivalent rates. An example for the latter is given in Fig. 5. ${ }^{9}$

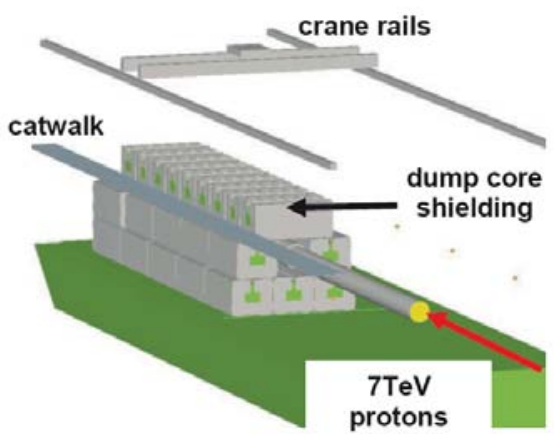

Fig. 6 FLUKA geometry of a LHC beam dump. For clarity of the representation the walls of the cavern are not shown. ${ }^{11,12)}$

\section{Beam Dump Study}

The two LHC beam dumps (one for each counter-rotating beam, see Fig. 1) consist of air-cooled graphite cores which are surrounded by iron shielding. For the latter, old magnet yokes filled with concrete were used. In order to avoid structural damage of the cores the beams are swept with a circular shape over the front faces of the dumps. This ensures the integrity of the core which is also controlled with beam loss monitors installed inside of the shielding. Nevertheless, should a core degrade it has to be replaced which is evidently a delicate intervention as the interior of the shielding is highly radioactive.

Thus, the core exchange has been studied during the design phase with FLUKA simulations. ${ }^{11,12)}$ The geometry included a very realistic representation of the dump structures in which the magnet yokes, as well as the air gaps in between them, were modeled in great detail. Figure 6 shows a three dimensional view of this geometry.

For the estimation of doses received by personnel during the core replacement maps of residual ambient dose equivalent rate were computed assuming one year of operation (233 fills with $2.6 \times 10^{14}$ protons dumped after each fill) and several cooling periods after the last beam dump. The exchange consists of several steps, such as disconnecting the core from the upstream beam pipe, removing the top-layer of the shielding with the overhead crane and lifting of the broken core assembly. During the work personnel is exposed to residual radiation also directly from the core and from the inside of the shielding. Thus, residual dose rates were calculated for different configurations, among others, with closed and open shielding. Figure 7 presents results for one week of cooling time. ${ }^{11,12)}$ The dose rate maps then served as basis for the estimation of job doses, including a detailed break-down in work steps which allowed modification or optimization of those steps which contribute most to the received dose. In this context, dry-runs performed during installation proved to be indispensable for obtaining realistic scenarios and time estimates. $^{13)}$

The beam dump core exchange is only one example where FLUKA has been used to consider the ALARA (As Low As Reasonably Achievable) principle already during 


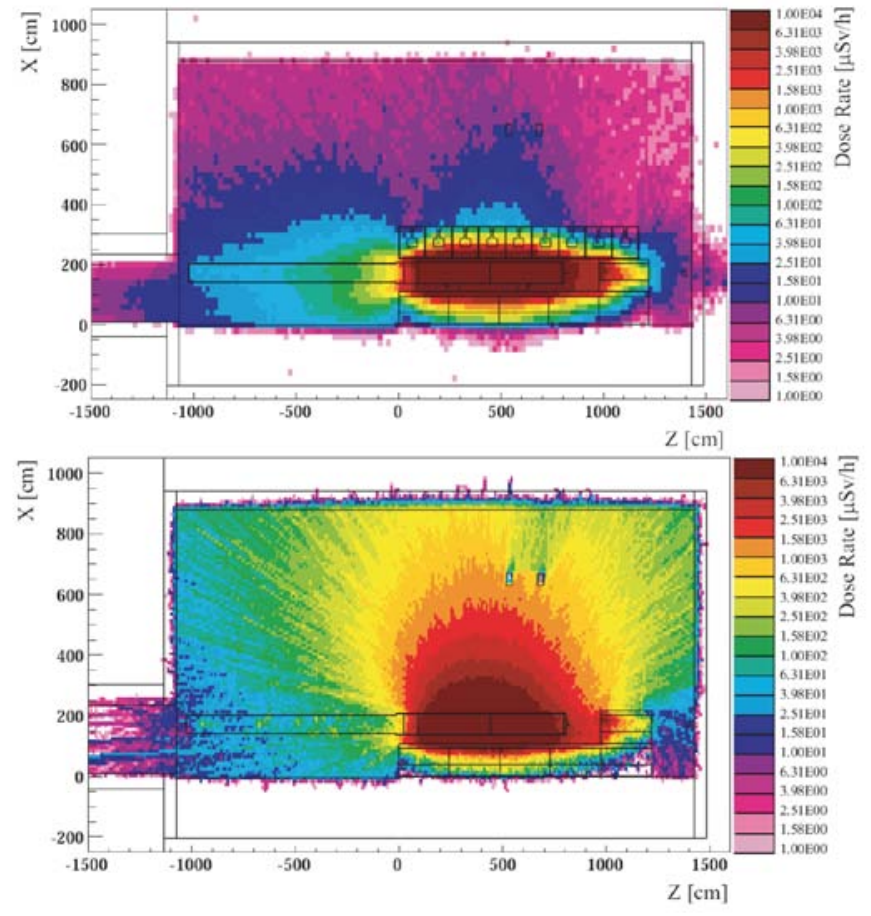

Fig. 7 Spatial distributions of ambient dose equivalent rate after one year of operation and one week of cooling with the top shielding in place (top) and removed (bottom). Results are shown for a vertical section through the centre of the dump core. $^{11,12)}$

LHC design. Since related design modifications ensure considerable cost-savings during operation, e.g., due to shorter accelerator down-times, this method is now routinely applied also to other CERN accelerators and in the design of LHC upgrades.

\section{Radiation Detector Responses}

\section{Response Measurements and Calculations}

Stray radiation fields around the LHC consist of a large range of particle types and energies. Most relevant for radiation protection considerations are neutrons, protons, charged pions and electromagnetic particles; their energies can extend from those of thermal neutrons to several tens of $\mathrm{GeV}$. The monitoring of workplaces has to be adapted accordingly which also includes monitor calibration. For the latter the use of standard sources may not be appropriate and additional studies were required. The studies comprised comprehensive FLUKA calculations of energy- and particle type-dependent monitor responses, their verification with mono-energetic particle beams as well as measurements at the above mentioned CERF facility.

As an example, Fig. 8 shows the FLUKA geometry of a high-pressure IG5 ionization chamber which is installed to monitor the radiation levels in the counting rooms of the LHC experiments. Depending on the expected radiation field two filling gas types are used: argon if charged particles contribute significantly to the prompt dose equivalent and hydrogen if the field is dominated by neutrons. For both gas types response functions were calculated with FLUKA for

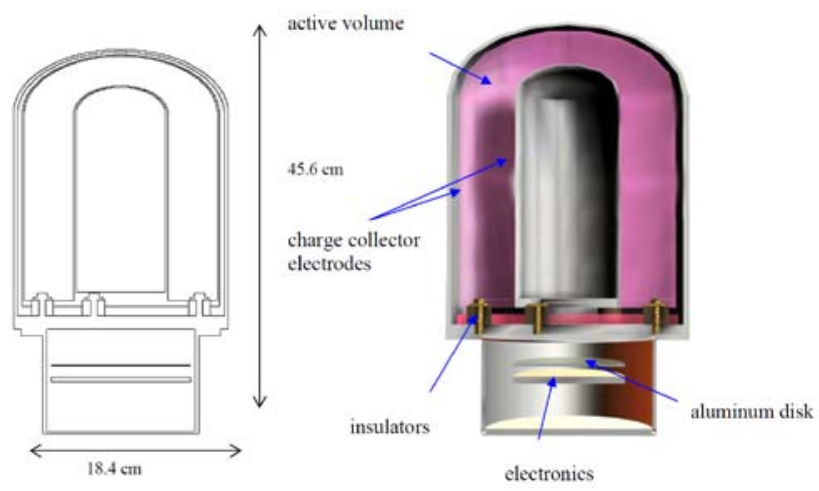

Fig. 8 Geometry of an IG5 ionisation chamber as used in the FLUKA simulations. ${ }^{14)}$

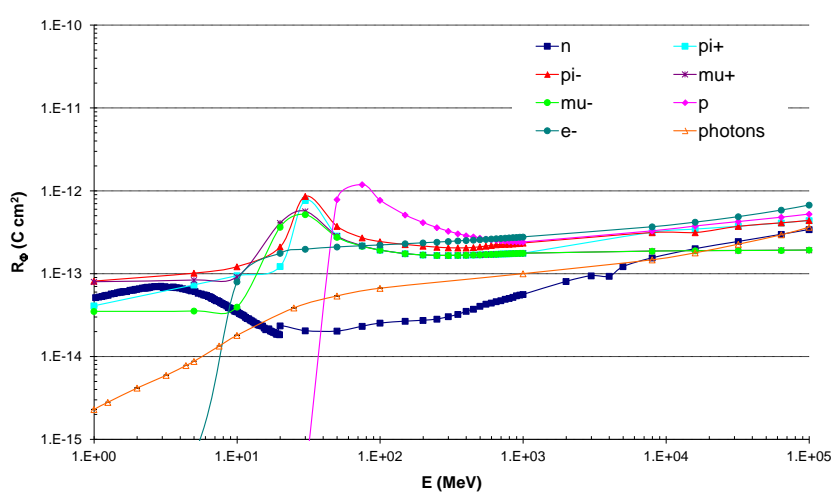

Fig. 9 Response functions for protons, neutrons, muons, charged pions, photons and electrons expressed in terms of created charge per unit fluence for a hydrogen-filled IG5 chamber. Calculated values are connected using a constrained cubic spline fit. ${ }^{15)}$

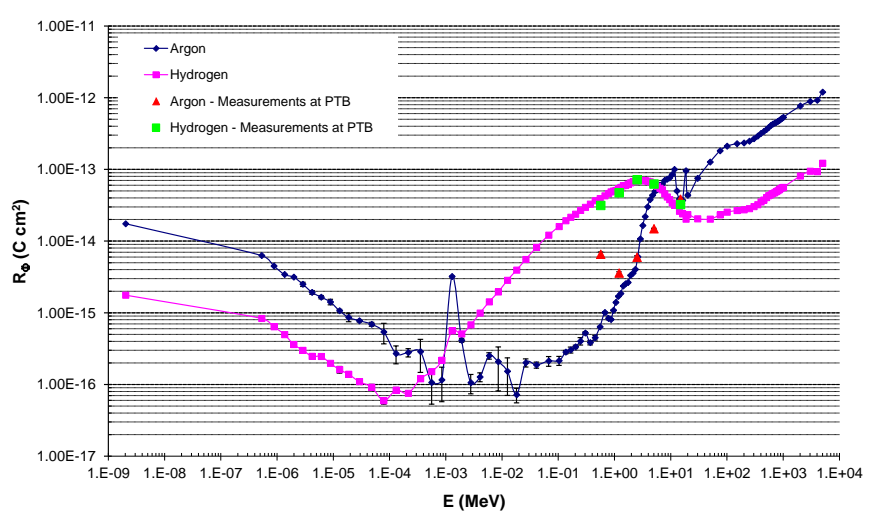

Fig. 10 Neutron response expressed in terms of created charge per unit fluence for argon- and hydrogen-filled IG5 chambers. FLUKA results are shown together with experimental data obtained in mono-energetic neutron beams. ${ }^{14)}$

the most common particle types from eV to $\mathrm{GeV}$ energies in different irradiation configurations. The functions for a hydrogen-filled chamber above $1 \mathrm{MeV}$ are presented in Fig. 9. ${ }^{15)}$ The results for neutrons agree well with experimental data obtained in mono-energetic beams as shown in Fig. 10. ${ }^{14)}$ 


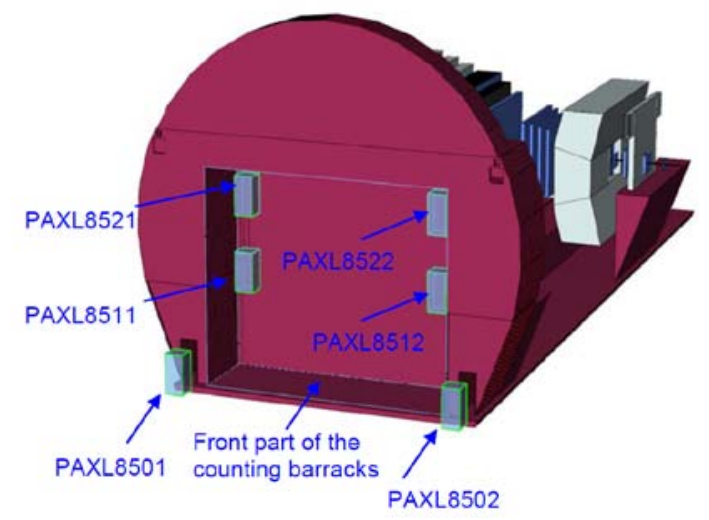

Fig. 11 Locations of the IG5 ionization chambers which monitor the radiation level in the counting room area of the LHCb experiment. The LHCb detector can be seen in the background. ${ }^{15)}$

\section{Field Calibration Study}

Hydrogen-filled IG5 ionization chambers monitor the radiation fields in many underground workplaces of the LHC, among others also in the counting room area of the LHCb experiment. As previously outlined, standard calibration sources may not represent well enough the actual field characteristics. Hence, ambient dose equivalent as well as particle energy spectra were calculated with FLUKA for the different monitor locations in the LHCb counting room area (see Fig. 11).

Folding the latter with the calculated response functions (Fig. 9) gives the charge collected by the respective monitor per proton-proton collision. The ratio of dose equivalent and collected charge in a stray radiation field is commonly referred to as "field calibration coefficient" and can be compared to calibration coefficients for standard sources, such as ${ }^{238} \mathrm{PuBe}$. The comparison for the detectors in the LHCb counting rooms is shown in Fig. 12, where differences of up to $25 \%$ are observed. The field calibration coefficients differ also among each other by up to $20 \%$ due to the specific field characteristics at the different monitor locations. Consequently, safety margins of up to $25 \%$ have to be considered when setting alarm levels for the different monitors unless the calculated field calibration coefficients are directly entered into the software of the monitoring system.

\section{Conclusion}

The FLUKA code is an integral part of all radiation protection studies for the LHC. It is the most suitable code of its kind as it provides unique features, such as combined prompt and residual dose rate calculations and the simulation of proton and heavy ion collisions at LHC energies. All physics models in FLUKA are individually benchmarked against experimental data and a wealth of benchmark studies for applications exists. Furthermore, relatively little freedom in model selection by the user along with default settings for most of the free parameters allows a high level of quality

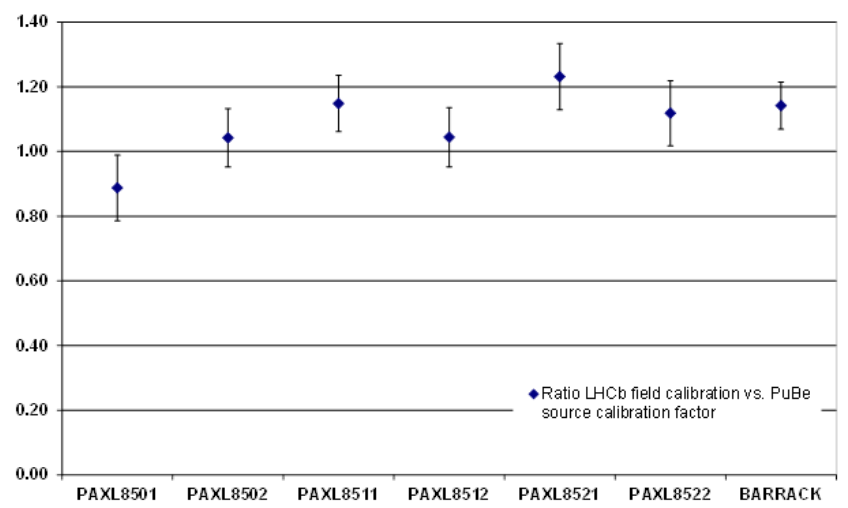

Fig. 12 Ratios of the field calibration coefficients determined in the mixed field at LHCb to the ${ }^{238} \mathrm{Pu}$-Be source-based calibration coefficient. ${ }^{15)}$

assurance which is indispensable for radiation protection applications.

Although complex FLUKA simulations require a significant amount of time (especially for the preparation of the input) many LHC-related applications have proven that this investment during the design phase can lead to considerable savings at a later stage, for example when accelerator operation profits from a well-optimized handling of activated components. Realistic simulations also allow a reduction of safety margins which translates directly into cost savings, e.g., for shielding.

Finally it should be noted that the development of many new FLUKA features and model improvements related to radiation protection applications for the LHC were driven by requirements in this field. It underlines the importance of model development and benchmarking as part of state-of-the-art radiation protection.

\section{References}

1) A. Fassò, A. Ferrari, J. Ranft, P.R. Sala, FLUKA: a multi-particle transport code, CERN-2005-10 (2005), INFN/TC_05/11, SLAC-R-773.

2) G. Battistoni, S. Muraro, P. R. Sala, F. Cerutti, A. Ferrari, S. Roesler, A. Fassò, J. Ranft, "The FLUKA code: Description and benchmarking," Proceedings of the Hadronic Shower Simulation Workshop 2006, Fermilab 6-8 September 2006, M. Albrow, R. Raja eds., AIP Conference Proceeding 896, 31-49 (2007).

3) S. Roesler, R. Engel, J. Ranft, "The Monte Carlo Event Generator DPMJET-III," Proceedings of the Monte Carlo 2000 Conference, Lisbon, 23-26 October 2000, A. Kling, F. Barao, M. Nakagawa, L. Tavora, P. Vaz (eds.), Springer-Verlag Berlin, 1033-1038 (2001).

4) M. Pelliccioni, "Overview of fluence-to-effective dose and fluence-to-ambient dose equivalent conversion coefficients for high energy radiation calculated using the FLUKA code," Radiat. Prot. Dosim., 88, 279-297 (2000).

5) H. Hirayama, "Inter-comparison of medium-energy neutron attenuation in iron and concrete (8)," Proceedings of the $10^{\text {th }}$ Meeting of the Task Force on Shielding Aspects of Accelerators, Targets and Irradiation Facilities, SATIF-10, CERN 2-4 June 2010, to be published. 
6) C. Adorisio, private communication (2010).

7) C. Theis, D. Forkel-Wirth, S. Roesler, H. Vincke, Ambient dose equivalent and field calibration studies for the final design of the LHCb shielding, Technical Note CERN-SC-2007-035-RP-TN (2008).

8) C. Theis, K. H. Buchegger, M. Brugger, D. Forkel-Wirth, S. Roesler, H. Vincke, "Interactive three-dimensional visualization and creation of geometries for Monte Carlo calculations," Nucl. Instr. Meth. Phys. Res., A562, 827-829 (2006).

9) M. Brugger, H. Khater, S. Mayer, A. Prinz, S. Roesler, L. Ulrici, H. Vincke, "Benchmark studies of induced radioactivity produced in LHC materials, Part II: remanent dose rates,” Radiat. Prot. Dosim., 116, 12-15 (2005).

10) M. Brugger, A. Ferrari, S. Roesler, L. Ulrici, "Validation of the FLUKA Monte Carlo code for predicting induced radioactivity at high-energy accelerators," Nucl. Instr. Meth. Phys. Res., A562, 814-818 (2006)

11) M. Brugger, A. Ferrari, D. Forkel-Wirth, M. Fürstner,
E. Lebbos, M. Lorenzo-Sentis, S.Roesler, V. Vlachoudis, J. Vollaire, "Predictions of residual dose rates with FLUKA for high energy accelerators at CERN," Proceedings of the $1^{\text {st }}$ Workshop on Accelerator Radiation Induced Activation, PSI, 13-17 October 2008, PSI Proceedings 09-01, 194-205 (2009).

12) J. Vollaire, D. Forkel-Wirth, S. Roesler, Calculation of remanent dose rate maps in the LHC beam dump caverns, Technical Note CERN-SC-2006-044-RP-TN (2007).

13) J. Vollaire, D. Forkel-Wirth, S. Roesler, Estimation of the individual and collective doses due to the replacement and transport of one of the LHC beam dump cores, Technical Note CERN-SC-2007-060-RP-TN (2007).

14) C. Theis, M. Rettig, S. Roesler, H. Vincke, Simulation and experimental verification of the response functions of Centronic high-pressure ionization chambers, Technical Note CERN-SC-2004-023-RP-TN (2004).

15) C. Theis, D. Forkel-Wirth, D. Lacarrere, S. Roesler, "Monte Carlo-based field calibration of radiation monitors for the Large Hadron Collider at CERN,” Nucl. Technol., 168, 713-718 (2009). 